\title{
Quantum correlations between a pair of Raman photons from a single atom under arbitrary excitation condition
}

\author{
Anil K. Patnaik, ${ }^{1,2}$ Girish S. Agarwal, ${ }^{3, *}$ C. H. Raymond Ooi, ${ }^{1,2}$ and Marlan O. Scully ${ }^{1,2,4}$ \\ ${ }^{1}$ Princeton Material Institute and Department of Mechanical and Aerospace Engineering, Princeton University, \\ Princeton, New Jersey 08544, USA \\ ${ }^{2}$ Institute for Quantum Studies and Department of Physics, Texas A and M University, Texas 77843, USA \\ ${ }^{3}$ Department of Physics, Oklahoma State University, Stillwater, Oklahoma 74078, USA \\ ${ }^{4}$ Max-Planck-Institute für Quantenoptik, Hans-Kopfermann-Strasse 1, D-85748, Garching, Germany \\ (Received 6 July 2005; published 17 October 2005; publisher error corrected 19 October 2005)
}

\begin{abstract}
The quantum correlation between a pair of Stokes and anti-Stokes photons, involving a Raman emission process is calculated. All realistic radiative and nonradiative decays are included in the calculation and the photon correlation between the pair for arbitrarily strong excitation fields and detunings are calculated. The correlation function shows photon antibunching, and a damped sinusoidal behavior with respect to the time delay between the measurement of the two photons. The current system can also produce four photon entanglement.
\end{abstract}

DOI: 10.1103/PhysRevA.72.043811

PACS number(s): 42.50.Dv, 42.50.Gy, 42.50.Ct, 32.80.-t

\section{INTRODUCTION}

The generation of high intensity correlated photon source is a subject of current interest. The Stokes-anti-Stokes Raman emission doublet (RED) of Fig. 1 is such a source. This has been shown recently in a series of elegant experiments by independent groups [1-5]. The application of such correlated pairs in quantum communications [1], quantum memory [3], single photon generation [2], and also controllable few-photon pulse generation [4] have been considered. Harris and co-workers have experimentally demonstrated the generation of counterpropagating paired photons with coherence times of about $50 \mathrm{~ns}$ and controllable waveforms [5]. It has been shown that such a pair could be used for enhancement of resolution in quantum microscopy and lithography [6]. Configurations, similar to the above, which generated RED pair were discussed in the early works of Agarwal and Jha [7] and Scully and Druhl [8].

In this paper we calculate the two-photon quantum correlation between the generated RED pair (see Fig. 1) using the master equation formulation [9] and Onsager-Lax regression theorem $[10,11]$. This method takes into account all the realistic radiative and nonradiative decays and works well for any arbitrary detunings and field strengths. Here we explicitly report the results of a possible experimental situation with a nonresonant pump excitation and a strong resonant anti-Stokes excitation. The present consideration corresponds to a small cell containing low density of $\mathrm{Rb}$ gas, such that reabsorption of the emitted photons and propagation effects are ignored. The ion traps would be another candidate for the observation of such effect [12].

The organization of the paper is as follows. In Sec. II, we present the formulation for the quantum correlation between the RED pair using the master equation and the quantum

*On leave from Physical Research Laboratory, Navrangpura, Ahmedabad 380 009, India regression theorem. In Sec. III, we present the numerical results for photon correlation and its manipulation in ${ }^{87} \mathrm{Rb}$ atomic system. We analytically calculate the correlation function under certain assumptions in Sec. IV and compare

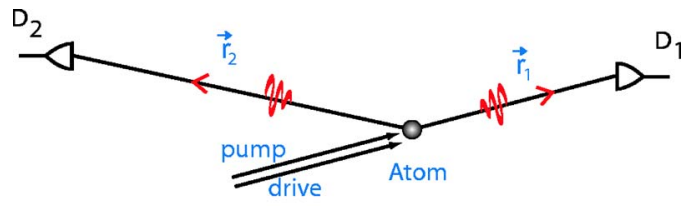

(A)

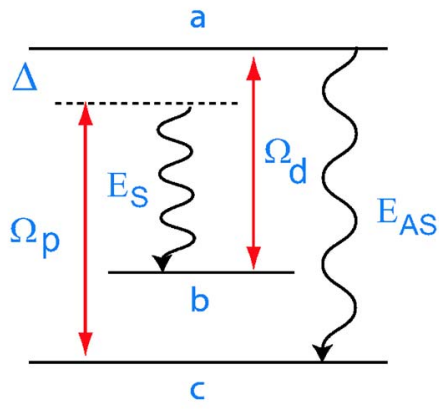

(B)

FIG. 1. (Color online) (a) A three level atom, when illuminated by a pump and drive laser, emits a pair of Stokes and antiStokes photon. Two detectors $D_{1}$ and $D_{2}$ detect the pair of emitted photons. (b) The level scheme under consideration for the generation of the correlated photon pairs. The atom is initially taken to be in the ground state $c$. The pump field $\Omega_{p}$, which is detuned from the $a \leftrightarrow c$ transition by $\Delta$, generates the Stokes photon $E_{S}$ and the driving field $\Omega_{d}$, that is on resonance with the $a \leftrightarrow b$ transition, drives the atom to state $a$ generating the anti-Stokes photon $E_{\mathrm{AS}}$. The energy level scheme corresponds to ${ }^{87} \mathrm{Rb}$ with $|a\rangle \equiv\left|5^{2} P_{1 / 2}, F=2\right\rangle, \quad|b\rangle$ $\equiv\left|5^{2} S_{1 / 2}, F=2\right\rangle$, and $|c\rangle \equiv\left|5^{2} S_{1 / 2}, F=1\right\rangle$. 
the result with the wave function approach $[6,14]$. Finally we summarize our results in Sec. V.

\section{THEORETICAL FORMULATION OF THE QUANTUM CORRELATIONS}

We consider a lambda system that corresponds to the hyperfine levels of the ${ }^{87} \mathrm{Rb}$ atom (see Fig. 1). Initially the atom is assumed to be in the ground state $|c\rangle$. A pump field $E_{p}$, when couples the $|c\rangle \leftrightarrow|a\rangle$ transition with a large detuning $\Delta$, a Stokes photon $E_{S}$ is emitted and the atom goes to state $|b\rangle$. Further the atom is driven by a resonant drive field $E_{d}$ that couples the $|b\rangle \leftrightarrow|a\rangle$ transition to generate the anti-Stokes photon $E_{\mathrm{AS}}$ in the $|b\rangle \leftrightarrow|a\rangle$ transition. We consider that two detectors $D_{1}$ and $D_{2}$, placed at $\vec{r}_{1}$ and $\vec{r}_{2}$, detect one photon each at two different times $t_{1}$ and $t_{2}$, respectively. The twophoton intensity correlation function $G^{(2)}$ is the coincidence of detection of these two photons at the two detectors. The simultaneous probability of detection of one photon at $D_{1}$ and the other at $D_{2}$, is given by

$$
\begin{aligned}
G^{(2)}\left(\vec{r}_{1}, t_{1} ; \vec{r}_{2}, t_{2}\right)= & \left\langle\hat{E}_{S}^{-}\left(\vec{r}_{1}, t_{1}\right) \hat{E}_{\mathrm{AS}}^{-}\left(\vec{r}_{2}, t_{2}\right) \hat{E}_{\mathrm{AS}}^{+}\left(\vec{r}_{2}, t_{2}\right) \hat{E}_{S}^{+}\left(\vec{r}_{1}, t_{1}\right)\right\rangle \\
& +(1 \leftrightarrow 2),
\end{aligned}
$$

the averaging is done over the initial field states at the detectors. Here, $\hat{E}_{F}^{ \pm}(F \rightarrow S, \mathrm{AS})$ are positive and negative frequency components of the field emitted by the system. The first term in the above equation corresponds to the simultaneous probability of detection of the Stokes photon clicking the detector $D_{1}$ at $t_{1}$ and the anti-Stokes clicking $D_{2}$ at $t_{2}$, and the second term corresponds to $D_{2}$ detecting the Stokes photon and $D_{1}$ detecting anti-Stokes.

The field of the generated pair of photons can be written in terms of their corresponding dipole oscillations at the source. In the far-zone approximation, the fields of the generated photons at the detector positions are given by the atomic dipole operators as [9],

$$
\begin{gathered}
\hat{E}_{S}^{+}\left(\vec{r}_{i}, t_{i}\right) \sim \hat{E}_{S_{0}}^{+}\left(\vec{r}_{i}, t_{i}\right)-\vec{\alpha}_{i} \hat{\sigma}_{b a}\left(t_{i}-r_{i} / c\right), \\
\hat{E}_{\mathrm{AS}}^{+}\left(\vec{r}_{i}, t_{i}\right) \sim \hat{E}_{\mathrm{AS}_{0}}^{+}\left(\vec{r}_{i}, t_{i}\right)-\vec{\beta}_{i} \hat{\sigma}_{c a}\left(t_{i}-r_{i} / c\right),
\end{gathered}
$$

where

$$
\begin{aligned}
& \vec{\alpha}_{i}=\left(\omega_{a b}^{2} / c^{2} r_{i}\right) \hat{r}_{i} \times \hat{r}_{i} \times \vec{\wp}_{a b}, \\
& \vec{\beta}_{i}=\left(\omega_{a c}^{2} / c^{2} r_{i}\right) \hat{r}_{i} \times \hat{r}_{i} \times \vec{\wp}_{a c},
\end{aligned}
$$

and $\hat{E}_{F_{0}}^{+}\left(\vec{r}_{i}, t_{i}\right)$ is the positive frequency component of the free field part of the emitted radiation at $\left(\vec{r}_{i}, t_{i}\right), \hat{\sigma}_{i j}$ is the transition operator $|i\rangle\langle j|$ that can be expressed in terms of the density operator $\hat{\rho}$ as $\left\langle\hat{\sigma}_{i j}\right\rangle \equiv \operatorname{Tr} \hat{\sigma}_{i j}(t) \hat{\rho} \equiv \rho_{j i}(t)$.

We assume that initially the field state at the detector is vacuum, i.e., $E_{F_{0}}^{ \pm}=0$. We substitute $\hat{E}_{F}^{ \pm}$into Eq. (1) to obtain the photon correlation function in terms of the atomic operators as

$$
\begin{aligned}
G^{(2)}\left(\vec{r}_{1}, t_{1} ; \vec{r}_{2}, t_{2}\right)= & \left|\alpha_{1}\right|^{2}\left|\beta_{2}\right|^{2}\left\langle\hat{\sigma}_{a b}\left(\tau_{1}\right) \hat{\sigma}_{a a}\left(\tau_{2}\right) \hat{\sigma}_{b a}\left(\tau_{1}\right)\right\rangle \\
& +(1 \leftrightarrow 2) .
\end{aligned}
$$

Here $\tau_{i}=t_{i}-r_{i} / c$ is the retarded time. We have used the transition operator property $\sigma_{i j} \sigma_{k l}=\delta_{j k} \sigma_{i l}$. It should be noted that the averaging has to be done over the dipole operators at different times. Such multitime averaging can be done using the Onsager-Lax regression theorem [10] which is stated as (see Refs. $[9,11]$ ): if $\hat{O}_{i}$ corresponds to a complete set of operators describing a system, and assuming that the behavior of the system is Markovian, if one-time mean value of an operator evolves as

$$
\left\langle\hat{O}_{m}\left(t^{\prime}\right)\right\rangle=\sum_{n} g_{m n}\left(t^{\prime}-t\right)\left\langle\hat{O}_{n}(t)\right\rangle, \quad \text { for } t^{\prime}>t,
$$

then the two-time correlation is given as

$$
\left\langle\hat{O}_{m}\left(t^{\prime}\right) \hat{O}_{l}(t)\right\rangle=\sum_{n} g_{m n}\left(t^{\prime}-t\right)\left\langle\hat{O}_{n}(t) \hat{O}_{l}(t)\right\rangle \Theta\left(t^{\prime}-t\right) .
$$

Here, $g_{m n}$ is a coefficient that depends on the difference of time $\left(t^{\prime}-t\right)$. The step function $\Theta(x)=1$ for $x>0$, and is zero otherwise.

The mean value of the transition operator evolution of the atomic scheme in Fig. 1 is governed by the standard density matrix equations for the three level lambda system

$$
\begin{aligned}
\frac{\partial \rho_{a a}}{\partial t}= & -2\left(\gamma_{a b}+\gamma_{a c}\right) \rho_{a a}+i \Omega_{d} \rho_{b a}-i \Omega_{d}^{*} \rho_{a b}+i \Omega_{p} \rho_{c a} \\
& -i \Omega_{p}^{*} \rho_{a c}, \\
\frac{\partial \rho_{a b}}{\partial t}= & -\left(\gamma_{a b}+\gamma_{a c}\right) \rho_{a b}-i \Omega_{d}\left(\rho_{a a}-\rho_{b b}\right)+i \Omega_{p} \rho_{c b}, \\
\frac{\partial \rho_{a c}}{\partial t}=- & \left(\gamma_{a b}+\gamma_{a c}+i \Delta\right) \rho_{a c}+i \Omega_{d} \rho_{b c}-i \Omega_{p}\left(\rho_{a a}-\rho_{c c}\right),
\end{aligned}
$$

$$
\frac{\partial \rho_{b b}}{\partial t}=2 \gamma_{a b} \rho_{a a}-i \Omega_{d} \rho_{b a}+i \Omega_{d}^{*} \rho_{a b}
$$

$$
\frac{\partial \rho_{b c}}{\partial t}=-\left(\Gamma_{b c}+i \Delta\right) \rho_{b c}+i \Omega_{d}^{*} \rho_{a c}-i \Omega_{p} \rho_{b a},
$$

$$
\frac{\partial \rho_{c c}}{\partial t}=2 \gamma_{a c} \rho_{a a}-i \Omega_{p} \rho_{c a}+i \Omega_{p}^{*} \rho_{a c} .
$$

Here $2 \gamma_{i j}$ are the decay rates from $|i\rangle \rightarrow|j\rangle$, and $\Gamma_{b c}$ is the dephasing rate of the Raman coherence. The above equation $\partial \rho / \partial t=L \rho$ has a formal solution

$$
\rho_{i j}(t)=\sum_{m n} f_{i j m n}(t) \rho_{m n}(0),
$$

where $f_{i j m n}=[\exp (L t)]_{i j m n}$. This equation gives the relation between the transition operators $\hat{\sigma}_{i j}$ at two different times. Equation (8) can be written in terms of $\hat{\sigma}_{i j}$ as 


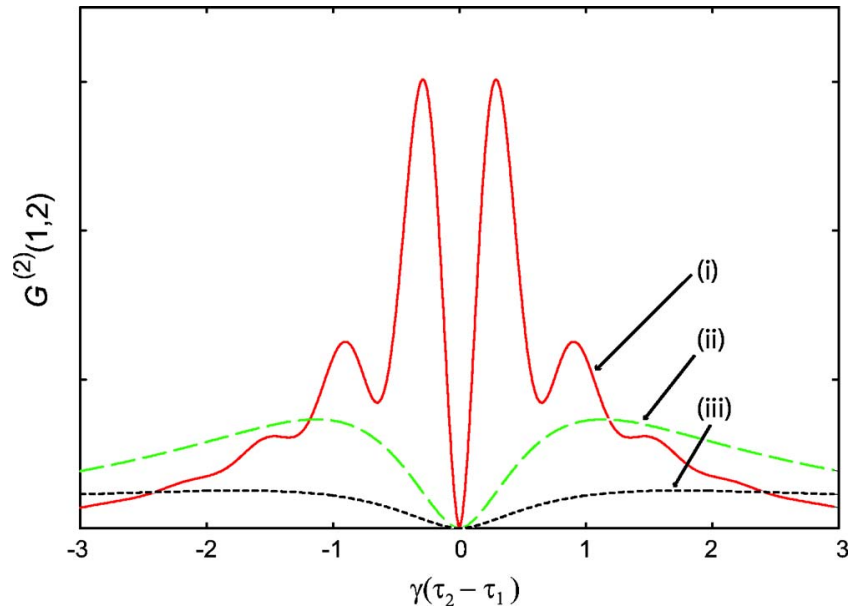

FIG. 2. (Color online) The two-photon correlation function as a function of delay between the two photons of the Raman pair. Here $\Omega_{d}=5 \gamma$ (i), $\gamma$ (ii), and $0.5 \gamma$ (iii), and $\Delta=10 \gamma, \Omega_{p}=0.1 \gamma, \gamma_{a c}=\gamma_{a b}$ $=\gamma$, and $\Gamma_{b c}=\gamma / 600$.

$$
\left\langle\hat{\sigma}_{j i}\left(\tau_{2}\right)\right\rangle=\sum_{m n} f_{i j m n}\left(\tau_{2}-\tau_{1}\right)\left\langle\hat{\sigma}_{n m}\left(\tau_{1}\right)\right\rangle, \quad \text { for } \tau_{2}>\tau_{1} .
$$

Therefore as stated in the regression theorem Eq. (6), the two time correlation function in Eq. (4) can be written as

$$
\begin{aligned}
G^{(2)}\left(\vec{r}_{1}, t_{1} ; \vec{r}_{2}, t_{2}\right)= & \left|\alpha_{1}\right|^{2}\left|\beta_{2}\right|^{2} \sum_{m n} f_{a a m n}\left(\tau_{2}-\tau_{1}\right) \\
& \times\left\langle\hat{\sigma}_{a b}\left(\tau_{1}\right) \hat{\sigma}_{n m}\left(\tau_{1}\right) \hat{\sigma}_{b a}\left(\tau_{1}\right)\right\rangle \\
& \times \Theta\left(\tau_{2}-\tau_{1}\right)+(1 \leftrightarrow 2) .
\end{aligned}
$$

Further using the operator algebra $\sigma_{i j} \sigma_{k l}=\delta_{j k} \sigma_{i l}$ the above equation reduces to

$$
\begin{aligned}
G^{(2)}\left(\vec{r}_{1}, t_{1} ; \vec{r}_{2}, t_{2}\right)= & \left|\alpha_{1}\right|^{2}\left|\beta_{2}\right|^{2} \sum_{m n} f_{a a m n}\left(\tau_{2}-\tau_{1}\right)\left\langle\hat{\sigma}_{a a}\left(\tau_{1}\right)\right\rangle \delta_{m b} \delta_{n b} \\
& \times \Theta\left(\tau_{2}-\tau_{1}\right)+(1 \leftrightarrow 2)=\left|\alpha_{1}\right|^{2}\left|\beta_{2}\right|^{2} f_{a a b b} \\
& \times\left(\tau_{2}-\tau_{1}\right)\left\langle\hat{\sigma}_{a a}\left(\tau_{1}\right)\right\rangle \Theta\left(\tau_{2}-\tau_{1}\right)+(1 \leftrightarrow 2) .
\end{aligned}
$$

The physical meaning of $f_{a a b b}$ is: probability of finding the atom in state $|a\rangle$ given that initially the atom was prepared in state $|b\rangle$. Note that once the Stokes photon is emitted, the atom makes a transition to $|b\rangle$. In order to produce the antiStokes photon, the system has to be excited to the state $|a\rangle$ that is represented by $f_{a a b b}$. And hence the two-photon correlation function $G^{(2)}$ is proportional to the term $f_{a a b b}$.

\section{NUMERICAL RESULTS}

The function $f_{a a b b}$ can be calculated from Eqs. (7), where all orders of the coherent fields are included. Thus the correlation function $G^{(2)}$ obtained in Eq. (11) holds for a vary broad range of parameters, e.g., one can choose any value of the field strength, detunings and spontaneous decay rates. For resonant intense pump and driving fields, the function $f_{a a b b}$ has been calculated analytically by Agarwal and Jha

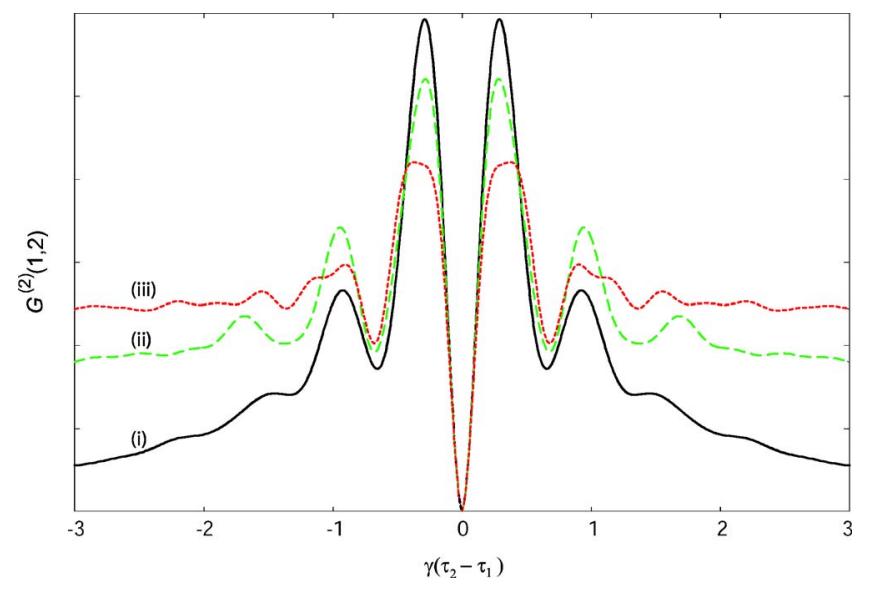

FIG. 3. (Color online) Numerical plot of the two-photon correlation function between the Raman pair in ${ }^{87} \mathrm{Rb}$ with strong pumping field. The curves correspond to $G^{(2)}$ with a fixed $\Omega_{d}=5 \gamma$ and different strengths of the pumping field $\Omega_{p}=1 \gamma$ (i), $3 \gamma$ (ii), and $5 \gamma$ (iii). All other parameters are same as in Fig. 2.

[7]. However, for the conditions of current experimental interest, we have to evaluate Eq. (11) numerically.

We have numerically calculated $G^{(2)}$ and plotted it in Fig. 2 for the given parameters of ${ }^{87} \mathrm{Rb}$. The spontaneous decay rates are $\gamma_{a b}=\gamma_{a c}=\gamma=2 \pi \times 3 \mathrm{MHz}$ and the ground state dephasing rate using a buffer gas could be $\Gamma_{b c} \approx 2 \pi$ $\times 5 \mathrm{kHz}[13]$. Here we have considered the case when $\Omega_{p}$ is very weak. In Fig. $2, G^{(2)}$ is zero at $\tau_{2}-\tau_{1}=0$; i.e., a zero count in the simultaneous measurement of the RED pairindicating the antibunching of the photons. This is because once the Stokes photon is emitted, the atom goes to the state $|b\rangle$ and needs to be pumped to the state $|a\rangle$ for emission of the anti-Stokes photon. Hence, the onset of the two-photon correlation depends on the strength of the driving field $\Omega_{d}$. Further, the frequency of oscillation in $G^{(2)}$ is also a function of the driving field. In fact, for $\Omega_{p}<\gamma$, the number of oscillations per each cycle of population, via $|c\rangle \rightarrow|b\rangle \rightarrow|a\rangle$ $\rightarrow|c\rangle$ path, is independent of pump field Rabi frequency $\Omega_{p}$. For higher strengths of the driving field $\Omega_{d}, G^{(2)}$ achieves a steady state after few oscillations of the atomic population in the $|a\rangle \leftrightarrow|b\rangle$ transition. The steady state is achieved due to the fact that all the spontaneous decays in the system are taken into account in the calculation. The number of cycles of the population and also the steady state value of $G^{(2)}$ depends on the strength of $\Omega_{p}$. For very small $\Omega_{p}$, the steady state value is zero. Note that for $\left(\tau_{2}-\tau_{1}\right) \sim$ integer multiples of $\gamma^{-1} / 2$, the minima of $G^{(2)}$ occurs. But the minima never touch zero due to the nonzero width of the state $|a\rangle$, even for $\Omega_{d}>\gamma$. However, for $\Omega_{d} \gg \gamma$, these minima are close to zero (not shown here).

For completeness, we present numerical results of twophoton correlation due to strong pumping field $\Omega_{p}$ in Fig. 3 . It should be noted that due to strong pumping field, the system cycles through $c \rightarrow b \rightarrow a \rightarrow c$ multiple times emitting a series of Stokes and anti-Stokes photons. Thus Fig. 3 depicts the correlation between the first Stokes photon and successive anti-Stokes photons emitted in each cycle, unlike Fig. 2 that corresponds to the correlation between a single pair of Stokes and anti-Stokes photon. Moreover, an increase in the 
intensity of the pumping field also increases the probability of populating the excited state $a$ via $c \rightarrow a$ transition. Thus for stronger $\Omega_{p}$, the frequency of oscillation in $G^{(2)}$ is not only due to drive field (unlike in Fig. 2) but also a function of the pumping field Rabi frequency. And due to various decay channels from $a, G^{(2)}$ ends up in a nonzero steady state value for longer time delay between observations. It may be noted that the intense pump produces multiple photon pairs. This system can thus be used to produce four photon entanglement which is being currently produced by optical parametric oscillators [15]. Howover, our system may find applications where we want to produce narrow bandwidth photon pairs.

\section{ANALYTICAL RESULTS IN THE LIMIT OF NEGLIGIBLE $\gamma_{a b}$}

It is interesting to study the system when $\gamma_{a b}$ is very small $\left(\gamma_{a b} \approx 0\right)$. Under this approximation, we can obtain an approximate analytical solution for $G^{(2)}$ that will help to understand the underlying physical phenomena. We also compare our result with the $G^{(2)}$ calculations reported using the wave function method and Weisskopff-Wigner approximation $[6,14]$.

We use the Laplace transform method to calculate the function $f_{a a b b}$ by solving the coupled Eqs. (7). In the Laplace domain, the Eqs. (7) reduce to a set of coupled algebraic equations. Assuming that the pumping field $\Omega_{p}$ is weak and the ground state coherence $\rho_{b c}$ is negligible, the function $f_{a a b b}$ in the Laplace transformed frame reduces to

$$
\begin{aligned}
\tilde{f}_{a a b b}(s)= & 2\left|\Omega_{d}\right|^{2} A /\left\{\left[s+2\left(\gamma_{a b}+\gamma_{a c}\right)\right] A B+2\left|\Omega_{d}\right|^{2}\left(s-2 \gamma_{a b}\right) A\right. \\
& \left.+2\left|\Omega_{p}\right|^{2}\left(s+\gamma_{a b}+\gamma_{a c}\right)\left(s-2 \gamma_{a c}\right) B\right\},
\end{aligned}
$$

where

$$
\begin{gathered}
A=2\left|\Omega_{p}\right|^{2}\left(s+\gamma_{a b}+\gamma_{a c}\right)+s\left(s+\gamma_{a b}+\gamma_{a c}\right)^{2}+s \Delta^{2}, \\
B=2\left|\Omega_{d}\right|^{2}+s^{2}+s\left(\gamma_{a b}+\gamma_{a c}\right) .
\end{gathered}
$$

The inverse Laplace transform gives the time evolution of $f_{a a b b}(t)$. However, since the denominator of $\tilde{f}_{a a b b}$ is a sixth order polynomial, it is nontrivial to get all the poles required for transforming back to $t$ space. Here $t=\tau_{2}-\tau_{1}$ is the time delay between the measurement of the two photons. Assuming $\gamma_{a b} \approx 0, \Omega_{p} \approx 0$ and the ground state dephasing $\Gamma_{b c}=0$, we obtain a simplified result in the original $t$ space

$$
\begin{aligned}
f_{a a b b}(t)= & \frac{2\left|\Omega_{d}\right|^{2} e^{-\gamma_{a c} t}}{4\left|\Omega_{d}\right|^{2}-\gamma_{a c}^{2}}\left[1-\frac{1}{2}\left(\exp \left[\sqrt{\gamma_{a c}^{2}-4\left|\Omega_{d}\right|^{2}} t\right]\right.\right. \\
& \left.\left.+\exp \left[-\sqrt{\gamma_{a c}^{2}-4\left|\Omega_{d}\right|^{2}} t\right]\right)\right] .
\end{aligned}
$$

Substituting the above Eq. (14) in $G^{(2)}$ into Eq. (11) we get

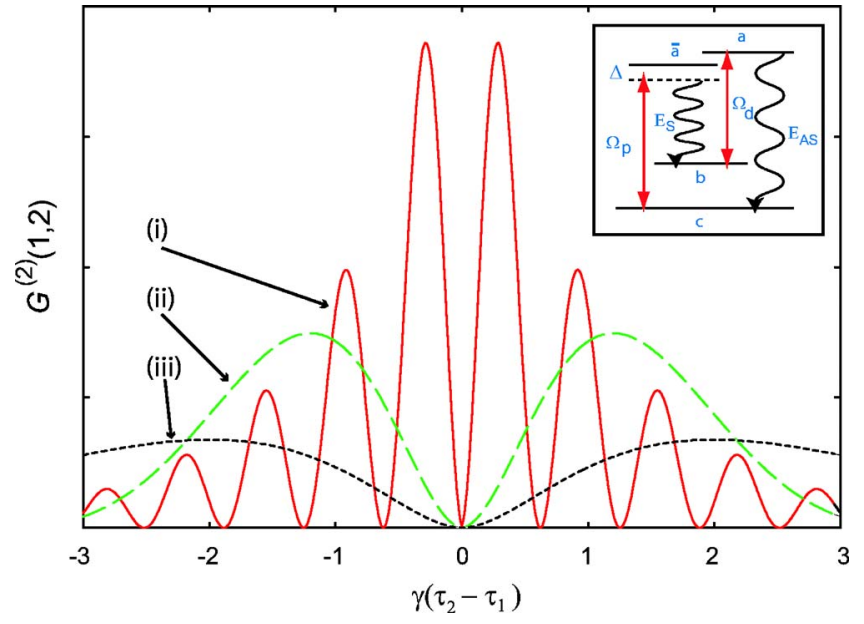

FIG. 4. (Color online) The two-photon correlation function as a function of delay between the two photons of the Raman pair. Here $\gamma_{a c}=\gamma, \Gamma_{b c}=0$, and $\gamma_{a b}=0.01 \gamma$. All other parameters are same as in Fig. 2.

$$
\begin{aligned}
G^{(2)}= & \left|\alpha_{1}\right|^{2}\left|\beta_{2}\right|^{2} \frac{2\left|\Omega_{d}\right|^{2} e^{-\gamma_{a c} t}}{4\left|\Omega_{d}\right|^{2}-\gamma_{a c}^{2}}\left\langle\sigma_{a a}\left(\tau_{1}\right)\right\rangle \\
& \left.\times\left(e^{\sqrt{\gamma_{a c}^{2}-4\left|\Omega_{d}\right|^{2} t}}+e^{-\sqrt{\gamma_{a c}^{2}-4\left|\Omega_{d}\right|^{2}} t}\right)\right] .
\end{aligned}
$$

Clearly, $G^{(2)}$ has two distinct regimes with different behavior depending on $2 \Omega_{d}>\gamma_{a c}$ (under damping) and $2 \Omega_{d}<\gamma_{a c}$ (over damping). In the earlier case $G^{(2)}$ oscillates with a frequency $\sqrt{4\left|\Omega_{d}\right|^{2}-\gamma_{a c}^{2}}$ and in the later case no oscillation is observed. This matches well with the numerical plot in Fig. 4. For $2 \Omega_{d}>\gamma_{a c}$, the above equation reduces to

$$
G^{(2)}=\eta \frac{\left|\Omega_{d}\right|^{2}}{\widetilde{\Omega}^{2}} e^{-\gamma_{a c}\left(\tau_{2}-\tau_{1}\right)} \sin ^{2}\left[\widetilde{\Omega}\left(\tau_{2}-\tau_{1}\right)\right] \Theta\left(\tau_{2}-\tau_{1}\right)+1 \leftrightarrow 2 .
$$

Here $\widetilde{\Omega}=\sqrt{\Omega_{d}^{2}-\gamma_{a c}^{2} / 4}$ and $\eta=\left|\alpha_{1}\right|^{2}\left|\beta_{2}\right|^{2}\left\langle\hat{\sigma}_{a a}\left(\tau_{1}\right)\right\rangle$. Clearly $G^{(2)}$ has an antibunching feature, i.e., it has zero count in the simultaneous measurement of both photons. The assumption used to obtain this analytical result $\gamma_{a b} \approx 0$ would mean that probability of emission of the Stokes photon is very small. However, a realistic system could be a four-level doublelambda scheme, as shown in the inset of Fig. 4, that contains an additional upper level-such that $\gamma_{a b} \approx 0$ but not $\gamma_{\bar{a} b}$. We have obtained $f_{a a b b}$ analytically for the four level scheme as well and the result is similar to that of the three level system presented here. We will discuss the details elsewhere.

Moreover, except for some constant numerical factors, the correlation function (16) is exactly the same as in Refs. $[6,14]$ where $G^{(2)}$ was obtained via a very different approach—solving the Schrödinger equation. This excellent agreement between these two completely different methods can be traced back to the same underlying approximation used in both the methods.

Further it is observed that for a weak $\Omega_{p}$, the population in the atom goes through only one cycle and hence $G^{(2)}=0$ for large $\left(\tau_{2}-\tau_{1}\right)$, as in Fig. 2. However, due to small $\gamma_{a b}$, the 
incoherent transfer of population from $|a\rangle \rightarrow|b\rangle$ is small. Hence the $G^{(2)}$ shows a characteristic Rabi oscillation with the minimum of each oscillation touching zero, see Fig. 4.

\section{SUMMARY}

We have calculated the two-photon intensity correlation between a pairs of Stokes and anti-Stokes photons, involving a sequential Raman emission process, using the density matrix equation and Onsager-Lax regression theorem. We have demonstrated that this method works for a very broad range of parameters of the excitation fields. We have shown that the correlation function shows photon antibunching feature, and it also shows sinusoidal behavior with respect to time delay between the measurements of the two photons. The frequency of oscillation depends on the coupling fields present in the system. The minimum in the observed oscillation depends on the decay rate of the excited state. We have also obtained an analytical result for a limiting case of very weak Stokes emission.

\section{ACKNOWLEDGMENTS}

We thank V. A. Sautenkov and A. Muthukrishnan for useful discussions. We also thank DARPA, ONR (Grant No. N00014-03-1-0639, N00014-04-1-0336), AFOSR (Grant No. F49620-01-1-0566), and Welch Foundation (Grant No. A-1261) for support.
[1] A. Kuzmich, W. P. Bowen, A. D. Boozer, A. Boca, C. W. Chou, L.-M. Duan, and H. J. Kimble, Nature (London) 423, 731 (2003).

[2] C. W. Chou, S. V. Polyakov, A. Kuzmich, and H. J. Kimble, Phys. Rev. Lett. 92, 213601 (2004).

[3] C. H. van der Wal, M. D. EIsaman, A. André, R. L. Walsworth, D. F. Phillips, A. S. Zibrov, and M. D. Lukin, Science 301, 196 (2003).

[4] M. D. Eisaman, L. Childress, A. André, F. Massou, A. S. Zibrov, and M. D. Lukin, Phys. Rev. Lett. 93, 233602 (2004).

[5] V. Balic, D. A. Braje, P. Kolchin, G. Y. Yin, and S. E. Harris, Phys. Rev. Lett. 94, 183601 (2005).

[6] M. O. Scully and C. H. R. Ooi, J. Opt. B: Quantum Semiclassical Opt. 6, S816 (2004); for the enhancement of resolution in other two-photon sources, see M. D’Angelo, M. V. Chekhova, and Y. Shih, Phys. Rev. Lett. 87, 013602 (2001); A. N. Boto, P. Kok, D. S. Abrams, S. L. Braunstein, C. P. Williams, and J. P. Dowling, Phys. Rev. Lett. 85, 2733 (2000); U. W. Rathe and M. O. Sculy, Lett. Math. Phys. 34, 297 (1995).

[7] G. S. Agarwal and S. S. Jha, Z. Phys. B 35, 391 (1979).
[8] M. O. Scully and K. Drühl, Phys. Rev. A 25, 2208 (1982).

[9] G. S. Agarwal, Quantum Statistical Theories of Spontaneous Emission and Their Relation to Other Approaches, Springer Tracts in Modern Physics: Quantum Optics (Springer-Verlag, Berlin, 1974).

[10] L. Onsager, Phys. Rev. 37, 405 (1931); M. Lax, in Stastitical Physics, edited by M. Chretien, E. P. Gross, and S. Deser (Gordon and Breach, New York, 1966), p. 403.

[11] M. O. Scully and M. S. Zubairy, Quantum Optics (Cambridge University Press, Cambridge, 1997), p. 300.

[12] F. Diedrich and H. Walther, Phys. Rev. Lett. 58, 203 (1987).

[13] V. A. Sautenkov, M. M. Kash, V. L. Velichansky, and G. R. Welch, Laser Phys. 9, 1 (1999).

[14] C. H. R. Ooi, A. K. Patnaik, and M. O. Scully Noise and Information in Nanoelectronics, Sensors, and Standards III, Proc. of SPIE 5846, 1 (2005).

[15] M. Eibl, S. Gaertner, M. Bourennane, C. Kurtsiefer, M. Zukowski, and H. Weinfurter, Phys. Rev. Lett. 90, 200403 (2003); P. Trojek, C. Schmid, M. Bourennane, H. Weinfurter, and C. Kurtsiefer, Opt. Express 12, 276 (2004). 